\title{
Application of Intelligent Transportation Systems (ITS) for Regional Development: Case Studies in Integrated Functional Approach
}

\author{
Koorosh Gharehbaghi ${ }^{1}$, Neville Hurst ${ }^{1, a}$ and Kathryn Robson ${ }^{1}$ \\ ${ }^{1}$ RMIT University, Melbourne, Australia
}

\begin{abstract}
This paper evaluates the application of Intelligent Transportation Systems (ITS) specifically as a part of regional development. Although ITS has been extensively studied in the context of city-wide developments, research on such developments for the regional enhancement is scarce. Importantly, transportation development for regional areas differs to that of traditional urban provinces. Whilst the transportation developments in urban areas need to concentrate on a range of diverse community requirements, for regional areas, such population are less diverse and more alike. Consequently, developed ITS in the regional areas needs to concentrate on more homogeneous communities and their needs. There are many factors influencing regional transportation developments, however functionality is one of the most critical aspects. Although functionality is an important aspect of all developments, it is considered to be the most crucial factor for regional areas. Fittingly, this paper will examine some of the most important transportation functionality issues for regional areas. To further explore this theme, the surrounding areas of Fukushima and Tokyo were studied to highlight their regional transportation requirements. These two case studies were then compared using factor analysis method. The comparison generally found that, due to its geographical location Fukushima aligned its regional functionalities more broadly. Further, this paper proposes a streamlined approach to analyze the successful application of transportation development in the Fukushima and Tokyo regional areas. To do so, an integrated functional approach was carefully undertaken. The proposed approach specifically focused on incorporating mobility and ITS as the key ingredients for the regional developments. It was found that, such integrated functional approach would therefore increase innovation and productivity as the result of successful application of ITS for regional development.
\end{abstract}

\section{Introduction}

As highlighted by Andres et al., (2016) [1] the expansion of transportation infrastructure is an essential component for the successful development of rural and regional communities. Bullock et al., (2011) [2] together with Wasson et al., (2011) [3] highlighted that an important attribute of Intelligent Transportation Systems (ITS) is the ability of such technological advancements for both urban and regional areas. Further, key aspects of transportation infrastructure for the regional areas encompass:

- Ensuring effective interconnection of remote areas [4]. This includes embracing and interconnecting the rural communities ensure that all regional transportation issues are vigilantly considered.

- Proficiently utilizing the appropriate ITS as the basis of regional development [5]. Although, the inclusion of ITS is typically aligned with urban transport, as a part of the regional transportation considerations, ITS could also provide important advantages.

- Successfully linking regional communities to urban areas for improved accessibility. Perhaps the most important aspect of transportation infrastructure for regional areas is the inclusion of effective accessibility [6]. Generally speaking, transportation accessibility requires both short and long-term considerations [7]. While short-term considerations include the convenience of transportation accessibility, long-term deliberation includes encompassing foreseeable regional growth and developments.

- Warranting long-term planning and appropriate enduring investment. This comprises implementation of various planning initiatives for rural communities. Here, transportation planning includes the consideration of rural environment and subsequent surrounding as the basis of the regional transportation development.

Appropriately, developed ITS in regional areas needs to concentrate in a more homogeneous manner towards community and its needs. Although other authors including Shu and Yao-Jan, (2013) [8] along Bhaskar and Chung (2013) [9] among many, emphasized the additional ITS abilities for the regional developments, more study is required. Accordingly, this paper aims to

\footnotetext{
${ }^{a}$ Corresponding author: neville.hurst@rmit.edu.au
} 
review the application of ITS for regional development and in doing so examines a number of case studies.

\section{Literature review}

As already conversed, transportation development for regional areas differs to that of traditional urban areas. Whilst transportation developments in urban areas need to concentrate on a range of diverse community requirements, for regional areas, such communities are less diverse [10, 11]. As Cao and Orru (2014) [12] highlighted, the most important transportation accessibility issues in regional areas include functionality together with performance. However, to further validate such hypothesis, a closer examination of the regional transportation functionality is required.

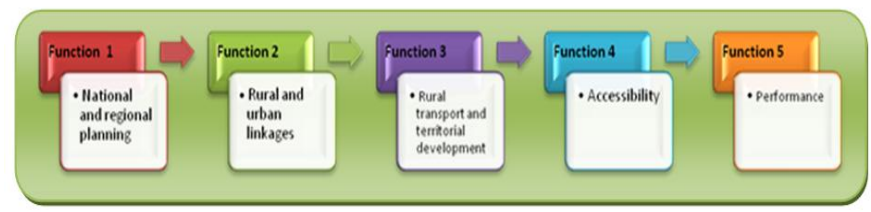

Figure 1: the regional transportation functionality.

It can be observed from Figure 1; regional transportation functionality consists of five main components:

1. National and regional planning, which includes a cluster rather than individual development $[13,14]$. The jurisdictional concerns may be difficult; however, this approach is necessary to bring about absolute regional amplification.

2. Rural and urban linkages. The alignment and connecting of both the rural and urban transportation systems is an important strategy [15]. Such strategy ensures that the regional transportation systems are not neglected, but rather cared for.

3. Rural transport and territorial development. The development of regional areas is highly dependent upon adequate transportation [16]. Insufficient rural transport not only obscures any regional development, but also limits territorial growth.

4. Accessibility. Of all the main functionalities, accessibility is perhaps the most challenging component $[17,18]$. This is due to the remoteness of some locations. The regional transportation accessibility maybe somewhat inadequate and/or not insufficient.

5. Performance. Of all the main functionalities, performance is the most immensely deliberated $[19,20]$. For the regional areas in particular, performance of the transportation services could undeniably make or break any future proposal and spending.

Accordingly, as the basis of ITS for regional development, this paper considers these five functions as the foundation of an integrated approach. To fully understand such integration, the transportation development for regional areas need to be carefully reviewed. Figure 2 articulates the asymmetry of regional transportation developments with the inclusion of ITS.

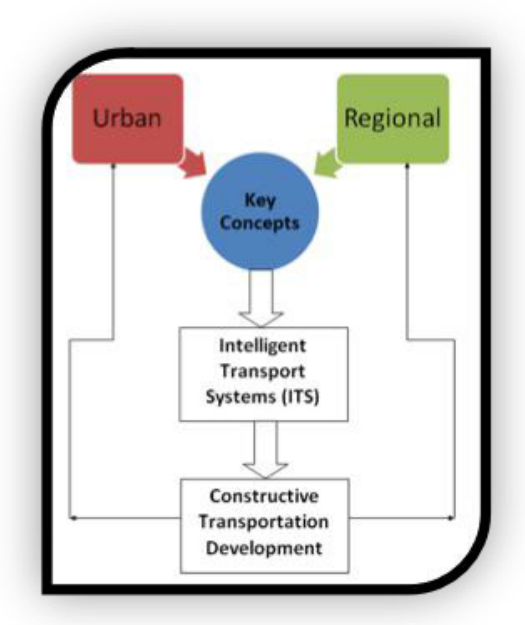

Figure 2: asymmetrical of regional transportation developments.

The proposed asymmetry is skewed towards the urban rather than rural development. Such orientation is due to a greater focus upon ITS in urban areas [21, 22]. Nevertheless, Gudmundsson et al., (2016) [23] highlighted that the importance of transportation for regional development be categorized into the following areas: a) Freight management, b) Road safety and security, c) Traffic management, d) Environment protection, e) Public transport, f) Automotive telematics, g) Parking management and h) Road user charging. Appropriately, these categories are represented in Figure 3 .

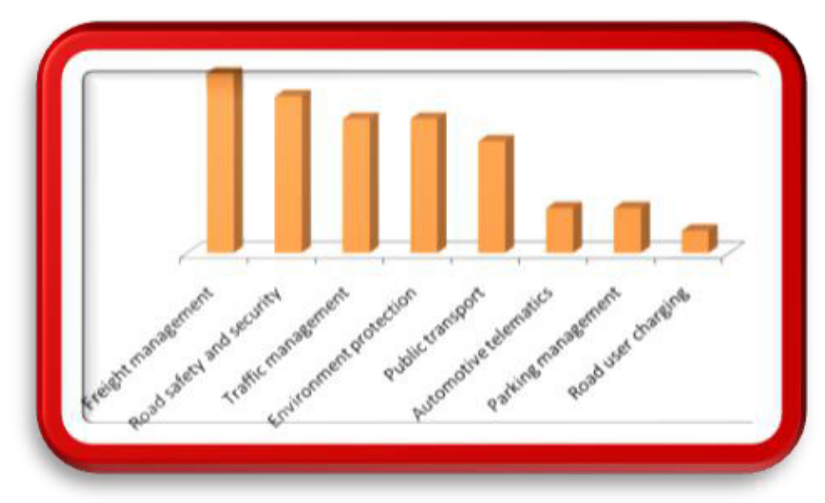

Figure 3: Transportation development for regional areas.

Figure 3 symbolizes the main areas of the regional development. The analyses provided here, is based on reviewed literature and a number of interpreted case studies. Hardisty (2010) [24] highlighted freight management as of absolute importance for the regional development, whilst Thekdi and Lambert (2012) [25] claimed that the road user charging has the least significance. Such analysis could be due to a small number of road users within the regional areas.

On one hand, road safety and security will be the second most important transportation factor for any regional developments [26]. On the other, Kahraman and Cevik Onar (2015) [27], reasoned that the effective traffic management along with environmental protections 
possess lesser effects than the previous two transportation factors. While it can be worked out that, due to lesser demand than the urban areas, public transport, automotive telematics and parking managements are the succeeding regional transportation issues (in that order). Subsequently, to fully demonstrate the importance of the ITS as a part of transportation development for the regional areas, a integrated functional approach is indispensable.

\subsection{ITS for the regional development: Integrated functional approach}

Conventionally, ITS can be defined as an amalgam of various methods, such as computational technologies, used to further enhance the overall functionality of all types of transport [27]. Such computational technologies integrate various traffic and transit management techniques as a part of overall functional enhancement approach and includes superior accessibility [28]. Nevertheless, to further ensure the successful application of transportation development for the regional areas, an integrated functional approach need to be carefully executed [26]. Such alignment will specifically focus on incorporating mobility and ITS as key ingredients for regional developments.

ITS also utilizes various methods such as computational technologies to further enhance the overall functionality of all types of transport [29]. Such computational technologies integrate various traffic and transit management techniques as a part of overall ITS functional enhancement approach including superior accessibility [30].

Although ITS has been extensively studied in the context of city-wide developments, research on ITS as part of regional enhancement is scarce. Importantly, transportation development for regional areas differs to that of traditional urban areas [31]. Whilst transportation developments in urban areas need to concentrate on a range of diverse community requirements, for regional areas, such communities are less diverse and more alike. Accordingly, developed ITS in regional areas needs to concentrate on a more homogeneous community and its needs [25]. On one hand, the concept of urbanization, which brings increased demand for the regional areas, needs to be carefully considered [32]. On the other, such regional development needs to prudently position, the competing drivers of engineering, environmental, economic and social. Subsequently, these factors then need to be integrated holistically, that is based on functional approach. This is presented in Figure 4.

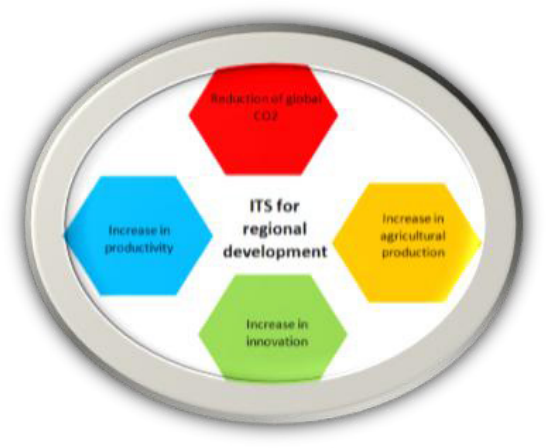

Figure 4: Integrated functional approach for regional development.

As identified, at the centre of this the integrated functional approach, lies the ITS. In other words, ITS is the essential element of this approach. Nonetheless, there are also four other important elements which complete this process. Using advanced transportation systems, could ultimately reduce the $\mathrm{CO}_{2}$ production through innovative emissions control management. Subsequently, the inclusion of ITS specifically for regional development, would also benefit from such advancement.

On one hand, increase in innovation can dramatically reduce losses and thus improve production and productivity rate. While, this will ultimately lead to better long-term revenue and thus increase economical benefits [33]. In addition, a successful application of ITS for regional development, requires a cautious alignment of transportation functionality and integrated functional approach. To investigate such theory, the following section (case studies) has been developed.

\section{Research methodology}

To further explore the alignment of 'transportation functionality' and 'integrated functional approach', the regional areas of Fukushima and Tokyo were studied. Such investigation would also carefully highlight the regional transportation requirements. These case studies evaluate the adaptation of ITS as a part of successful regional development and in doing so assess their regional transportation functionalities.

To effectively compare the transportation development for regional areas, the five main components of regional transportation functionality (see section 2.0) are compared on the Fukushima and Tokyo rural zones. It is important to highlight that such analysis was assisted by reports produced by 2012 Citylab together with 2017 Ministry of LITT (Land, Infrastructure, Transport and Tourism). These concentrated and inclusive reports were carefully studied and interpreted to determine the key ITS variances for both cities' regional development. Moreover, factor analysis was then used as the basis of comparing these two case studies and their subsequent findings carefully discussed. 


\section{Case Studies}

\subsection{Fukushima}

Fukushima is the capital city of Fukushima prefecture in Japan. This city has a transportation service containing rail and bus systems. The city uses the Iizaka rail line and an extensive bus network, to serve its regions. Figure 5 represents the general topography of Fukushima and its regional rail transportation.

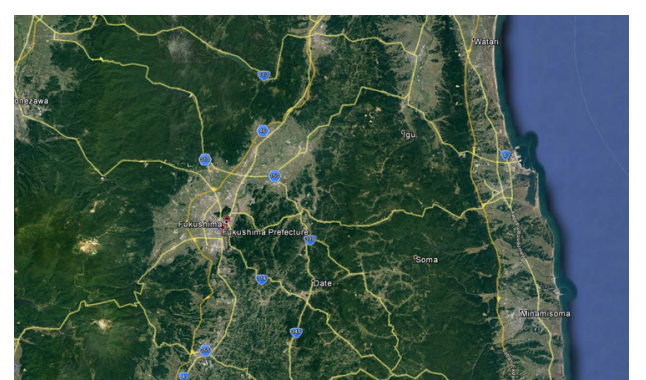

Figure 5: Topography of Fukushima and its regional rail transportation.

As it can be observed, Fukushima and its regional rail transportation is well spread. Such distribution, however, will also create ITS difficulties due to its scale. Not only does such ITS require significant funding but complication exists within such system integration.

\subsection{Tokyo}

Tokyo is the capital city of Japan and its greater area is the most populated metropolitan area in the world [34]. The transport network in Greater Tokyo includes public and private rail and highway networks; airports for international, domestic, and general aviation; buses; motorcycle delivery services, walking, bicycling, and commercial shipping. Moreover, the public transport systems within Greater Tokyo is serviced by 158 lines, 48 operators, $4,714.5 \mathrm{~km}$ of operational track and 2,210 stations [34]. Approximately, 40 million passengers use Tokyo's rail system daily (Citylab, 2017). Figure 6 represents the general topography of Tokyo and its regional rail transportation.

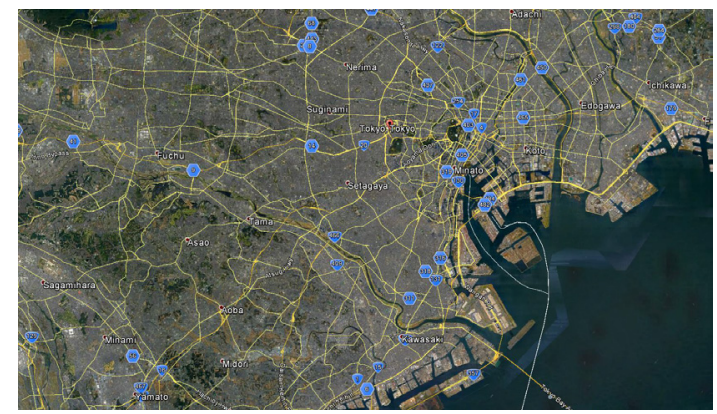

Figure 6, Topography of Tokyo and its regional rail transportation.

As it can noticed, Tokyo and its regional rail transportation is extremely spread. Such distribution will also significantly complicate the ITS. This is possibly due to many different system requirements, particularly dealing with functionality and overall performance.

\subsection{Case studies comparison}

In comparing the Fukushima and Tokyo case studies, specific regional transportation consideration needs to be measured. These considerations include:

- Foremost, the rural strategic alignment needs to be carefully deliberated. This consideration needs to cautiously contemplate not only land issues, but also alignment of all jurisdictional governance and any future national frameworks.

- The inclusion of innovative transport tools such as ITS, to thoroughly advance and integrate future regional developments.

Nevertheless, as already noted, the regional transportation functionality consists of five main components which are, National and regional planning; Rural and urban linkages; Rural transport and territorial development; Accessibility; and Performance.

Accordingly, to effectively compare the transportation development for regional areas, the five main components are contrasted on the Fukushima and Tokyo peri-urban zones. It is important to highlight that such analysis where assisted by reports produced by Citylab and Japan Ministry of Land, Infrastructure, Transport and Tourism [34]. Before commencing the functionalities analysis of the both cities, their trajectories of change were also explored. Figure 7 provides the projected transportation change for both cities via spatial presentation.

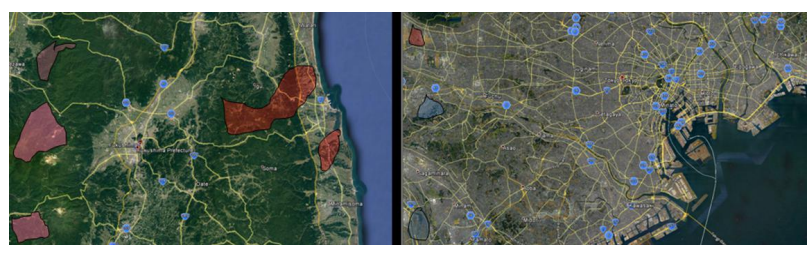

Figure 7: spatial representation of the projected transportation change for both cities.

As it can be summarized, for Fukushima, the projected transportation change is well spread with eastern suburbs having the most extreme (red) trajectories. This is most likely due to the 2011 nuclear disaster which mostly effected the eastern areas. Conversely, for Tokyo, the projected transportation change is generally low (blue) with extreme being planned for a small area. This is due to Tokyo's outstanding transportation infrastructure.

\subsubsection{Factor analysis}

Once the projected transportation of the both cities been briefly discussed, the actual functionalities analysis for the both metropolitans was then carried out. This comparison was based on the available data from reports produced by Citylab [35] and Japan Ministry of Land, Infrastructure, Transport and Tourism [34]. In addition, a factor analysis was used to characterize the correlations between the variables $X_{a}$ (for 5 main components and 4 functional approaches) of which the $X_{a i}$ (specific 
instances). The following conventional factor analysis model was thus used:

$$
F_{\chi \ldots}=\sum_{p} \ell_{a p} F_{p i}+\epsilon_{a i \ldots}
$$

To streamline the modeling process, a maximum value of 1.0 was used as the basis of total output. This output value was thus the basis of the regional functionalities and integrated functional approach factors. This analysis is presented in Table 1 .

Table 1, case studies comparison.

\begin{tabular}{|l|c|c|}
\hline \multicolumn{1}{|c}{ Regional functionalities } & Fukushima & Tokyo \\
\hline Accessibility & 0.213 & 0.236 \\
\hline National and regional planning & 0.219 & 0.111 \\
\hline Performance & 0.332 & 0.532 \\
\hline Rural and urban linkages & 0.127 & 0.045 \\
\hline Rural transport and territorial development & 0.109 & 0.076 \\
\hline \multicolumn{1}{|c|}{ Integrated functional approach } & Fukushima & Tokyo \\
\hline Increased in agricultural production & 0.136 & 0.09 \\
\hline Increased in innovation & 0.565 & 0.601 \\
\hline Increased in productivity & 0.167 & 0.203 \\
\hline Reduction of global Co 2 & 0.132 & 0.106 \\
\hline
\end{tabular}

Table 1 represents the summary of the factor analysis of the regional functionalities and integrated functional approach for both cities. A Mplus software was used to calculate the presented analysis. To further demonstrate the findings Figures $8 \mathrm{a}$ and $8 \mathrm{~b}$ are also presented.

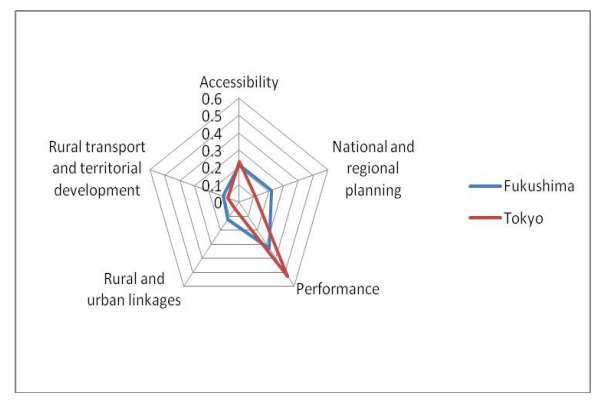

Figure 8a: 'regional functionalities' comparison.

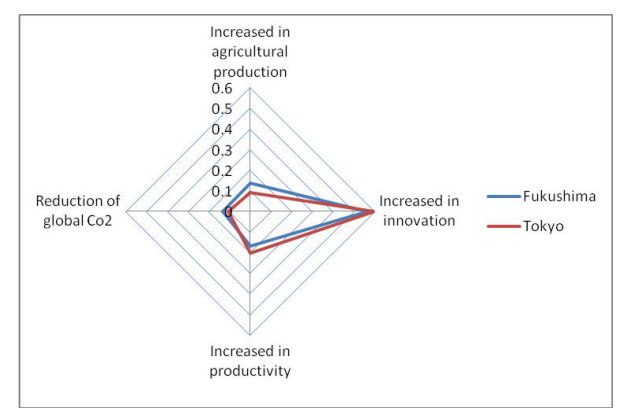

Figure 8b: 'integrated functional approach' comparison.

As it can be described, while Fukushima generally ranks lower in most areas, Tokyo seems to excel particularly in performance. Whilst Fukushima seems to be scoring higher on the national and regional planning along with rural and urban linkages; both cities score exceptional in increased innovation. Although, such outcome is likely due to Japan's reputation as a world technology leader; as a consequence of its geographical location, Fukushima needs to align its regional functionalities more broadly. Hence a higher national and regional planning along with rural and urban linkages score is observed.

For both cities, increase in innovation is extreme. This is considered a likely result of Japanese national and international efforts in research and development. Moreover, Tokyo mainly uses the most advanced and innovative technologies and thus is leading 'performance' not only with other Japanese cities but many cities around the globe. On the other hand, while for both cities and their regional areas, the reduction of global $\mathrm{CO}_{2}$ is seemingly comparable. However, the increase in agricultural production is slightly higher in Fukushima. This is due to Fukushima's vast available area dedicated specifically for agricultural industry.

Overall, the Japanese federal government continuously places significant importance for both areas of the nation. This is evident by the adaptation and utilization of ground-breaking ITS for both cities, but specifically Tokyo. Such outcomes would benefit Tokyo's transportation authority's allowing them to further improve and optimize the functionality of their regional transportation services.

\section{Lessons to be learned}

The comparison of the integrated functional approach for both Fukushima and Tokyo, further highlights the essential aspect of such an approach, specifically for the regional development. The successful implementation of Fukushima and Tokyo integrated functional approach highlights the significance of increased innovation and productivity as the result of successful application of ITS for regional development. Additionally, the case studies highlight such enhanced regional development specifically requires a cautious and gradual alignment of transportation functionality and integrated functional approach.

From the four integrated functional approaches for regional development, the inclusion of ITS specifically for regional development, requires developing and utilizing specific technologies that meet not only the nation, but also region's specific shortfalls. To improve such regional development, increasing innovation is highly desirable. Such innovation would not only increase the novelty of specific processes, but also lead to improve regional production and productivity rate. Additionally, such an improvement of integrated functional approach will also lead to supporting national advancements. 


\section{Conclusion}

This research found the inclusion of ITS is paramount for a successful transportation functionality and this in turn is necessary for successful regional development. In addition, such ITS adaptation also requires an absolute integrated functional approach. To demonstrate such proclamation, this paper utilized the regional areas of Fukushima and Tokyo as case studies. To contrast these two cities and their regional area developments, their actual transportation system functionalities were succinctly reviewed. While the Fukushima's vast available area is ideal for the agricultural production, it is Tokyo, which sets benchmarks in particular as the basis of both increased in innovation and productivity. Due to Tokyo's national and international significance, this city clearly outperformed Fukushima for both innovation and productivity.

Nevertheless, as this paper illustrated, the inclusion of integrated functional approach is a dominant factor for any regional development. Such integration needs to focus on more homogeneous communities along with their needs. Such integration needs to focus on more homogeneous communities along with their needs. Without such consideration, successful application of ITS for regional development is detrimentally affected. Finally, as this paper highlighted, one great challenge to achieve effective regional development is the cautious alignment of transportation functionality and integrated functional approach.

\section{Data Availability Statement}

Some or all data, models, or code generated or used during the study are available from the corresponding author by request.

\section{References}

1. S. Hernandez, A. Monzon, R. De Ona, "Urban Transport Interchanges: A Methodology for Evaluating Perceived Quality." Transportation Research, Part A 84, pp.31-43 (2016)

2. J. Wasson, G. Boruff, A. Hainen, S. Remias, E. Hulme, G. Farnsworth and D. Bullock, "Evaluation of Spatial and Temporal Speed Limit Compliance in Highway Work Zones". Transportation Research Record: Journal of the Transportation Research Board 2258, (2258), pp 1-15 (2011)

3. D. Bullock, M. Martchouk, F. Mannering, "Analysis of Freeway Travel Time Variability Using Bluetooth Detection". Journal of Transportation Engineering, 137 (10), pp 697-704 (2011)

4. K. Gharehbaghi and K. Robson, 'The Exploitation of ICT in Optimization of PTS: Productivity and Performance Enhancement Methods', in Proceedings of the 11th International Conference on Computer Modeling and Simulation, Association for Computing Machinery, Melbourne, 16 - 18 January, pp. 180-183. (2019)
5. K. Gharehbaghi, I. Clarkson, N. Hurst and F. Rahmani, "Transportation development for regional infrastructure: Implications for Australian rural areas", Transportation Research Procedia (2020) in press.

6. R. Kummitha and N. Crutzen, "How do we understand smart cities? An evolutionary perspective", Cities, 67, pp 43-52 (2017)

7. K. Gharehbaghi, and K. McManus, "TIS condition monitoring using ANN integration: an overview", Journal of Engineering, Design and Technology, 17, (1), pp. 204-217 (2019)

8. Y. Shu and W. Yao-Jan, "Travel Mode Identification Using Bluetooth Technology", Journal of Intelligent Transportation Systems, DOI: 10.1080/15472450.2017.1384698 (2013)

9. A. Bhaskar and E. Chung, "Fundamental understanding on the use of Bluetooth scanner as a complementary transport data", Transportation Research Part C: Emerging Technologies, 37, pp.42-72 (2013)

10. T. Krasemann, "Computational decision-support for railway traffic management and associated configuration challenges: An experimental study", Journal of Rail Transport Planning and Management, 5, (3) pp 95-109 (2015)

11. J. Mathey, S. Rößler, J. Banse and I. Lehmann, , "Brownfields As an Element of Green Infrastructure for Implementing Ecosystem Services into Urban Areas", Journal of urban planning and development, 141, (3) (2015)

12. Giacomo Cao and Roberto Orrù, "Current Environmental Issues and Challenges", Springer, pp 39-46 (2014)

13. J. Black, A. Paez and P. Suthanaya, "Sustainable Urban Transportation: Performance Indicators and Some Analytical Approaches", Journal of urban planning and development, 128, (4) (2002)

14. T. Kim, "Integrated Urban Systems Modeling: Theory and Applications", Springer, pp 191-199. (2012),

15. Gharehbaghi, K., McManus K., and Robson, K., (2019), "Minimizing the environmental impacts of mega infrastructure projects: Australian public transport perspective", Journal of Engineering, Design and Technology, in press.

16. E. Boschmann and M. Kwan, "Toward Socially Sustainable Urban Transportation: Progress and Potentials", international journal of sustainable transportation, 2, (3) pp 138-157 (2008)

17. K. Gharehbaghi and M. Georgy, "Utilization of Infrastructure Gateway System (IGS) as a Transportation Infrastructure Optimization Tool", International Journal of Traffic and Transportation Engineering, 4 (1), pp. 8-15 (2015)

18. K. Gharehbaghi and M. Myers, "Intelligent System Intricacies: Safety, Security and Risk Management Apprehensions of ITS", Proceedings of the 8th International Conference on Industrial Technology 
and Management, IEEE, Cambridge, United Kingdom, United Kingdom, pp 37-40. (2019)

19. K. Gharehbaghi and F. Rahmani "Deterioration of Transportation Infrastructures: Corrosion of Reinforcements in Concrete Structures", Materials and Technologies in Engineering, 940, pp 160 - 166 (2018)

20. K. Gharehbaghi and Farnes "Buried Transportation Structures: Fuzzy-Markov Computation Case Study", Proceedings of the 10th International Structural Engineering and Construction Conference: Interdependence between Structural Engineering \& Construction Management, Chicago, Illinois, United States, pp 1-6 (2019)

21. S. Ghosh and T. Lee "Intelligent Transportation systems: smart and green infrastructure design", 2nd edition, Boca Raton, FL : CRC Press (2012)

22. K. Gharehbaghi, K. Robson and Rahmani "Mega Transportation Infrastructure: a sustainable foresight", Transportation Research Procedia (2020) in press

23. H. Gudmundsson, R. Hall, G. Marsden and J. Zietsman "Sustainable transportation : indicators, frameworks, and performance management", Springer (2016)

24. P. Hardisty, "Environmental and economic sustainability", CRC Press/Taylor and Francis (2010)

25. S. Thekdi and J. Lambert, "Decision analysis and risk models for land development affecting Infrastructure systems", Journal of Risk Analysis, July, 32 (7), pp 1253-1269 (2012)

26. A. Sladkowski and W. Pamula "Intelligent Transportation Systems - Problems and Perspectives", Springer (2015)

27. C. Kahraman and S. Cevik Onar, "Intelligent techniques in engineering management theory and application", Springer (2015)

28. K. Gharehbaghi and K. Farnes "Process automation in Intelligent Transportation System (ITS)", International Journal of Machine Learning and Computing, International Association of Computer Science and Information Technology, 8 (3), pp. 294-297 (2018)
29. K. Gharehbaghi, "Artificial Neural Network for Transportation Infrastructure Systems", MATEC web of conferences, pp. 1-5 (2016)

30. L. Mattsson and E. Jenelius, "Vulnerability and resilience of transport systems - A discussion of recent research", Transportation Research Part A: Policy and Practice, 81, pp 16-34 (2015)

31. P. Centobelli, R. Cerchione and E. Esposito, "Environmental sustainability in the service industry of transportation and logistics service providers: Systematic literature review and research direction", Transportation Research Part D: Transport and Environment, 53, pp 454-470 (2017)

32. A. May, "Urban Transport and Sustainability: The Key Challenges", International Journal of Sustainable Transportation, 7, (3), pp 170-185 (2012)

33. F. Corman, A. D'Ariano, A. Marra, D. Pacciarelli and M. Sama, "Integrating train scheduling and delay management in real-time railway traffic control", Transportation Research Part E: Logistics and Transportation Review, 105, pp 213-239 (2017)

34. Ministry of LITT, Japan Ministry of Land, Infrastructure, Transport and Tourism, White Paper on Land, Infrastructure, Transport and Tourism in Japan, http://www.mlit.go.jp/en/statistics/whitepaper-mlit-index.html [viewed on 17/10/2017] (2017)

35. Citylab, https://www.citylab.com/transportation/2012/05/sec ret-tokyos-rail-success/2044/ [viewed on 15/09/2017] 